\title{
Bicuspid and Unicuspid Aortic Valves: Different Phenotypes of the Same Disease? \\ Insight from the GenTAC Registry
}

Joseph M Krepp, MD, ${ }^{a}$ Mary J Roman, MD, ${ }^{b}$ Richard B Devereux, MD, ${ }^{\mathrm{b}}$ Adrienne Bruce, MD, ${ }^{\mathrm{a}}$

Siddharth K Prakash, MD, PhD, ${ }^{\mathrm{c}}$ Shaine A Morris, MD, ${ }^{\mathrm{d}}$ Dianna M Milewicz, ${ }^{\mathrm{c}}$ Kathryn W

Holmes, MD, ${ }^{\mathrm{e}}$ William Ravekes, MD, ${ }^{\mathrm{e}}$ Ralph V Shohet, $\mathrm{MD},{ }^{\mathrm{f}}$ Reed E Pyeritz, MD, PhD, ${ }^{\mathrm{g}}$

Cheryl L Maslen, PhD, ${ }^{\mathrm{h}}$ Barbara L Kroner, PhD,${ }^{\mathrm{i}}$ Kim A. Eagle, MD, ${ }^{\mathrm{j}}$ Liliana Preiss, MS ${ }^{\mathrm{i}}$,

GenTAC Investigators and Federico M Asch, $\mathrm{MD}^{\mathrm{a}}$, (see supplemental material for full list of

GentTAC investigators)

${ }^{\mathrm{a}}$ MedStar Health Research Institute

fThe Queen's Medical Center

${ }^{\mathrm{b}}$ Weill Medical College of Cornell University

${ }^{\mathrm{g}}$ University of Pennsylvania

${ }^{\mathrm{c}}$ University of Texas - Houston

${ }^{\mathrm{h}}$ Oregon Health and Sciences University

${ }^{\mathrm{d}}$ Baylor College of Medicine

${ }^{\mathrm{i}} \mathrm{RTI}$ International

${ }^{\mathrm{e}} \mathrm{Johns}$ Hopkins University

${ }^{\mathrm{j}}$ University of Michigan

NHLBI National Registry of Genetically Triggered Thoracic Aortic Aneurysms and

Cardiovascular Conditions (GenTAC) is supported under contracts HHSN268200648199C and

HHSN268201000048C with RTI International.

There are no author conflicts of interest to report.

Short Title: Analysis of Bicuspid and Unicuspid Valves

Word Count: 4266

Corresponding Author:

Federico M Asch, MD FASE, FACC

100 Irving St, NW. Suite EB 5123, Washington, DC. 20010

Phone: (202) 877-3792 Email: federico.asch@medstar.net

This is the author manuscript accepted for publication and has undergone full peer review but has not been through the copyediting, typesetting, pagination and proofreading process, which may lead to differences between this version and the Version record. Please cite this article as doi:10.1111/ chd.12520. 


\begin{abstract}
Background: Unicuspid aortic valve (UAV) is a rare disorder, often difficult to distinguish from bicuspid aortic valve (BAV). BAV and UAV share valve pathology such as the presence of a raphe, leaflet fusion, aortic stenosis, aortic regurgitation, and/or ascending aortic dilatation, but a comprehensive echocardiographic comparison of patients with UAV and BAV has not been previously performed.
\end{abstract}

Methods: We investigated UAV and BAV patients at an early stage of disease included in GenTAC, a national registry of genetically-related aortic aneurysms and associated cardiac conditions. Clinical and echocardiographic data from the GenTAC registry were compared between 17 patients with UAV and 17 matched-controls with BAV.

Results: Baseline characteristics including demographics, clinical findings including family history of BAV and aortic aneurysm/coarctation, and echocardiographic variables were similar between BAV and UAV patients; aortic stenosis was more common and more severe in patients with UAV. This was evidenced by higher mean and peak gradient, smaller aortic valve area and more advanced valvular degeneration (all $\mathrm{p}<0.05$ ). There were no significant differences in aortic dimensions, with a similar pattern of enlargement of the ascending aorta.

Conclusion: The similar baseline characteristics with more accelerated aortic valve degeneration and stenosis, suggests that UAV represents an extreme in the spectrum of BAV syndromes. Therefore, it is reasonable to consider application of recommendations for management of patients with BAV to those with the rarer UAV.

Key Words: Bicuspid aortic valve; Unicuspid aortic valve; Congenital heart disease 


\section{Introduction}

Unicuspid aortic valve (UAV) is a rare congenital disorder with a prevalence that ranges from $0.02 \%$ in the echocardiographic referral population to $4-6 \%$ in patients undergoing valve replacement for aortic stenosis. ${ }^{1,2,3}$ Bicuspid aortic valve (BAV) disease, on the other hand, is the most common congenital cardiac malformation with a prevalence of about $1-2 \%$ in the general population. ${ }^{4} \mathrm{UAV}$ and BAV have similar clinical presentations (aortic stenosis or regurgitation) but the associated pathologies of UAV seem to develop earlier and progress at a faster rate than BAV. ${ }^{1}$ Indeed, most case series of patients with UAV describe young individuals with advanced valvular disease presenting for valve replacement. UAV is characterized as having either an eccentric, unicommissural orifice or a pinhole-shaped acommissural opening presenting with severe stenosis at birth. ${ }^{1,5,6,7}$ Unicommissural valves have a larger effective orifice area than acommissural valves; however, both have a smaller, rounded free edge compared to a trileaflet aortic valve (TAV). ${ }^{6,8,9,10,11}$ This severely narrowed opening and predilection for accelerated calcification of the aortic valve results in more frequent and earlier onset of aortic stenosis than found in individuals with BAV or TAV. ${ }^{6,12,13}$ The more severe pathology that correlates with the lower number of cusps suggests that there may be a phenotypic continuum of similar disease spanning from BAV to UAV. ${ }^{14}$ Despite this hypothesized continuum, to our knowledge an echocardiographic and clinical analysis of patients with UAV and BAV has never been performed. Whether they represent a spectrum of the same disease or they are, indeed, different conditions have yet to be determined. Furthermore, the prevalence of aortopathies in the UAV has not yet been thoroughly examined.

We undertook a comprehensive phenotypic and clinical comparison of patients with UAV and BAV enrolled in the national registry of patients with Genetically-related Thoracic 
Aortic Aneurisms and related cardiac conditions (GenTAC). GenTAC represents a unique opportunity to compare these two populations, as patients were enrolled at different stages of the disease, including those early in the progression. Our hypothesis is that patients with UAV and BAV share valvular and non-valvular features suggesting a common developmental defect in cusp separation or outflow tract septation and that UAV may represent a phenotypic extreme of the BAV spectrum.

\section{Methods}

Patients in GenTAC were enrolled at 8 nationally recognized centers for management of aortic diseases related to genetic conditions (Johns Hopkins, Baltimore; Weill Cornell Medicine, New York; University of Pennsylvania, Philadelphia; Baylor Medical Center, Houston; University of Texas, Houston; Oregon Health \& Science University, Portland; Queen's Hospital, Honolulu; National Institutes of Aging, Baltimore). Patients were enrolled on the basis of a diagnosis of a condition with genetically-associated aortopathy (BAV was the most frequent enrollment diagnosis). The rationale and design of GenTAC have been previously described. ${ }^{15,16}$ The GenTAC Registry is co-sponsored by the National Heart Lung and Blood Institute and the National Institute of Arthritis and Musculoskeletal and Skin Diseases. Patients gave written informed consent, and enrollment centers transmitted comprehensive case report forms to the data coordinating center (RTI, Rockville MD).

All patients in the GenTAC Registry that were diagnosed with a UAV by echocardiogram were included. A matched-control group consisting of GenTAC patients with BAV was created on a 1:1 ratio. To best identify a control group, matching was done on the basis of three clinical variables known to have an impact in valvular hemodynamics and aortic 
enlargement with BAV cohorts (age, gender and body surface area (BSA)). For any given UAV patient, a control BAV individual was identified with an exact age and gender match, and with the closest BSA. Given the small number of cases with UAV (and therefore of the control cohort), there was no attempt to compare genetic similarities among groups.

Echocardiograms in GenTAC were obtained at the enrolling centers and submitted for analysis to a centralized imaging Core Lab (iCORE, MedStar Health Research Institute, Washington, DC). All echocardiograms were analyzed according to a protocol that was agreed upon by the imaging experts from the iCORE and the enrolling centers. The image analysis protocol has been previously described in detail. ${ }^{17}$ In brief, all echocardiograms were analyzed by a single echocardiographer, blinded to the enrollment diagnosis and any clinical information. Aortic measurements were performed at predetermined aortic locations at end systole using the inner edge to inner edge technique and are reported as absolute dimensions. BAV was diagnosed as a valve with two commissures and an oval, "football-like" opening (Figure 1, left panel). UAV was defined as a valve with one or zero commissures and a rounded, "soccer ball-like" opening (Figure 1, center panel). Aortic valve regurgitation and stenosis were evaluated according to current American Society of Echocardiography guidelines. ${ }^{18,19}$ AVA (aortic valve area) was calculated by using the continuity equation with peak left ventricular outflow tract and aortic valve velocities. AVA was indexed to body surface area to classify aortic stenosis as mild $\left(>0.85 \mathrm{~cm}^{2} / \mathrm{m}^{2}\right)$, moderate $\left(0.60-0.85 \mathrm{~cm}^{2} / \mathrm{m}^{2}\right)$, or severe $\left(<0.60 \mathrm{~cm}^{2} / \mathrm{m}^{2}\right)$. An aortic valve degeneration score for both UAV and BAV was calculated according to criteria described by Michelena et al. ${ }^{20}$ Valvular degeneration was evaluated by scoring three separate components including leaflet thickening, mobility and calcification as 0 (normal), 1 (mildly abnormal), 2 (moderately abnormal), or 3 (severely abnormal). The three individual scores were added 
together to generate a composite degeneration score with a potential maximum score of $9 . \quad A$ comprehensive echocardiographic and clinical comparison of the two phenotypic groups was subsequently performed.

Statistical analyses were performed at the GenTAC data-coordinating center (RTI) using SAS version 9.3 with a two-tailed $p<0.05$ considered statistically significant. Classification of aortic valve stenosis and aortic valve regurgitation were placed into one of three groups: none, mild, or moderate and severe. Data are presented as mean \pm standard deviation for continuous variables and as percentages for categorical variables. All continuous variables were compared using Student's t-tests while discrete variables were compared by Chi-square analysis or Fisher's exact test.

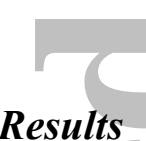

UAV was diagnosed in 17 GenTAC participants by echocardiography. BAV was diagnosed in over 600 patients, from which 17 were identified as matched controls based on gender, age and BSA for each of the UAV cases. The demographic and clinical characteristics of the subjects are described in Table 1. By study design, UAV and BAV groups were matched in mean age ( 15.4 years, range 2 months -47 years) and gender ( $71 \%$ male). Five patients in each group had either evidence of aortic coarctation by echocardiography or a history of coarctation repair; only one BAV patient had suffered an aortic dissection.

Interestingly, there were 3 patients in each group that had a family history of one or more of the following: BAV, aortic coarctation, or aortic aneurysm. Specifically, similar proportions of UAV and BAV patients had family histories of BAV (3 vs. 1), aortic coarctation (1 vs. 0) and aortic aneurysm (1 vs. 2). 
Echocardiographic analysis of UAV and BAV groups is shown in Table 2. There was no difference in aortic dimensions at any pre-specified level of the aorta. In both groups, the majority of patients had the classic phenotype of dilated ascending aorta described for BAV, characterized by the ascending aorta being larger than the sino-tubular junction (UAV 93.3\% vs. BAV 81.3\%, $\mathrm{p}=0.316$ ), which is demonstrated in Figure 1 (right panel). ${ }^{21}$ Interestingly, a finding of an ascending aorta being larger than the aortic root (at the sinuses of Valsalva) was seen more frequently in UAV than in BAV patients $(86.7 \%$ vs. $50 \%, \mathrm{p}=0.029)$.

On evaluation of valvular hemodynamics, there was no difference between the two groups with respect to the incidence and severity of aortic regurgitation. However, aortic stenosis was significantly more common in cases of UAV. Patients with UAV were more likely to have moderate or severe aortic stenosis than those with BAV $(58.8 \%$ vs. $17.6 \%, \mathrm{p}=0.011)$. Accordingly, UAV patients had significantly greater mean aortic valve gradients than patients with BAV (22.4 mmHg vs.10.4 mmHg, p<0.001) and greater peak aortic valve gradients $(39.2$ $\mathrm{mmHg}$ vs.18.6 mmHg, $\mathrm{p}<0.001)$. In addition to hemodynamic measures, there were significant differences between the two groups with respect to aortic valve degeneration as UAV subjects had significantly greater mean degeneration scores (2.8) than BAV subjects $(1.7, \mathrm{p}=0.043)$. Interestingly, the most notable difference between the two groups was seen in leaflet mobility characteristics as patients with UAV had mild-moderately reduced mobility (mean score 1.6) while BAV patients had only slightly abnormal leaflet mobility (mean score $0.7, \mathrm{p}<0.001$ ).

To further characterize patients with aortic stenosis and their degree of valvular degeneration, a subset analysis was performed in subjects found to have any degree of aortic stenosis (Table 3). Included in this analysis were 15 patients with UAV and 5 patients with BAV. Patients with UAV had more significant aortic stenosis than BAV patients (Indexed AVA 
$=0.67 \mathrm{~cm}^{2} / \mathrm{m}^{2}$ vs. $\left.0.90 \mathrm{~cm}^{2} / \mathrm{m}^{2}, \mathrm{p}=0.011\right)$. Although UAV patients with stenosis tended to be younger and have greater mean and peak aortic valve gradients than BAV patients, these differences were not statistically significant. Of note, patients in both groups had aortic stenosis despite having small degeneration scores with minimal calcification and thickening, a reflection of the young age and the restricted leaflet motion. Accordingly, the aortic valves of the UAV group had significantly decreased mobility compared to the BAV group (1.7 vs. $0.8, \mathrm{p}=0.005)$.

\section{Discussion}

Prior reports of UAV have included only data from postmortem or postsurgical specimens. ${ }^{2}$ This study is unique in that our population precedes significant aortic valve disease progression resulting in either death or surgical intervention to the aortic valve. We performed a comprehensive comparison of UAV and BAV patients that were age, gender, and BSA matched within the GenTAC Registry, to understand how similar (or dissimilar) these pathologies are. To our knowledge, this is the first study that combines echocardiographic and clinical evaluation of $\mathrm{UAV}$ and $\mathrm{BAV}$ in living patients not yet planning surgical valve replacement. Our findings are that: 1) demographic and clinical characteristics are similar; 2) family history of BAV and BAVassociated aortopathy was equally present in those with BAV and UAV; and 3) UAV patients have more degeneration of the aortic valve (mostly limited leaflet mobility) and therefore have a higher prevalence and severity of aortic stenosis.

It is interesting that the aortic dimensions, family history, and prior aortic surgeries were similar between the two groups. Furthermore, the majority of patients in both groups exhibited the typical pattern of dilated ascending aorta for BAV, postulated to be related to sheer stress on the ascending aorta due to turbulent flow through a narrowed aortic valve orifice. ${ }^{21,22}$ 
Intriguingly, the ascending aorta was larger than the aortic root more commonly in UAV than BAV, perhaps reflective of a more severe phenotypic pattern of the aortopathy or the hemodynamic consequences of more severe aortic stenosis. Patients from both populations had similar rates of aortic coarctation necessitating repair along with rates of surgical intervention at all pre-specified levels of the aorta. Similar to the pattern seen in BAV, UAV was also more common in males than females. ${ }^{12,14}$ Of note, a family history of BAV or of aortic aneurysm/dissection or coarctation was equally common in UAV and BAV individuals, suggesting a common, likely genetic, familial predisposition to these two forms of aortic valve pathology. Larger series of BAV patients have described a heritable pattern of BAV and the data from the present study suggests there is a common inheritance pattern in UAV patients. ${ }^{23}$ Given the lack of leaflet thickening and calcification, the degree of stenosis found in BAV and UAV is mostly due to differences in the cusp morphology impeding appropriate valve opening. The combination of these findings suggests that there is a common link in the inheritance patterns of patients with UAV and those with BAV.

To the extent that UAV and BAV share a common underlying predisposition, UAV appears to represent a more severe phenotype as patients with UAV tend to have earlier onset and faster progression of aortic stenosis, consistent with prior studies evaluating pathologic specimens of surgically excised aortic valves. ${ }^{1,2}$ Patients with UAV had smaller indexed aortic valve areas along with greater mean and peak aortic valve gradients than the BAV cohort, likely due to both the leaflet morphology as well as calcific progression. When analyzing the subset of patients from both groups diagnosed with aortic stenosis, UAV subjects had more severe aortic stenosis than BAV patients. Additionally, they tended to be younger than those with a BAV, 
although this was not statistically significant, possibly as a result of the relatively small sample size.

The mechanism for this pattern of aggressive aortic stenosis appears to be related mostly to leaflet mobility. Overall, patients with UAV had significant reduction of leaflet mobility whereas patients in the BAV cohort had only slightly reduced leaflet mobility, even when evaluating the subset of subjects with aortic stenosis. Both the presence and severity of aortic stenosis are most closely linked to reduced leaflet mobility, particularly in patients with UAV. One explanation for the low valve degeneration scores may be the young age of our patient population. In a prior study, Michelena et al. noted that BAV patients with valve degeneration had a mean age of 52 along with a relatively high prevalence of hypertension (43\%), both of which result in increased valve thickness and calcification. ${ }^{20}$ Aortic valves from the UAV and BAV groups in the present study did not demonstrate marked calcification or thickness given the young age of our study group (mean age 15.4 years). Additionally, much of the literature comparing BAV and UAV only includes excised valves from patients whose disease had progressed to either death or surgical intervention. ${ }^{2,24}$ These patients were older and represent a subset of the UAV and BAV population with advanced aortic valve disease and were far more likely to have significant valvular calcification and dystrophic aortic valves. While calcification of trileaflet aortic valves occurs naturally in patients after the age of 40 , valves become more dystrophic and more stenotic earlier in life in both UAV and BAV. ${ }^{24,25}$

Current recommendations for the management of patients with BAV are based on observations of large cohorts of patients. UAV, on the other hand, is a very rare condition and the observations are anecdotal in nature as most information about UAV has been gained from postmortem and post-surgical analysis. Demonstrating similarities between these two 
populations may allow adoption of current recommendations for management of BAV to patients with UAV with the understanding that aortic stenosis and aortic complications tend to occur earlier in those patients with UAV. Prior reports have demonstrated that patients with a more aggressively stenotic UAV manifest with early aortic involvement that is associated with an increased incidence of ascending dilatation, dissection and rupture. ${ }^{1,12,26}$ Owing to the earlier presentation and increased likelihood of more severe stenosis, UAV typically requires surgical treatment at least one to two decades earlier than BAV. ${ }^{8}$ Furthermore, with increasing patient age, differentiation between UAV and BAV can be challenging as focal calcification of UAV can mimic the appearance of the raphe seen in BAV on echocardiography. ${ }^{6,11}$ If cardiac imaging cannot distinguish between UAV and BAV then application of current BAV guidelines to these patients is an appropriate course of action with close monitoring for complications of both the aortic valve and aorta.

While our study has novel and interesting findings, some limitations must be acknowledged. The number of patients in the GenTAC registry with UAV was small, albeit larger than for any previous study. We were able to take advantage of the large number of subjects with BAV in the GenTAC cohort to identify a control group that was matched by the most significant demographic characteristics, therefore allowing a meaningful comparison of BAV and UAV patients. Unfortunately, the small number of cases also prevents a proper comparison of genetic variants, the ultimate method to prove the proposed linkage between BAV and UAV. Furthermore, the study likely did not capture UAV patients that required procedures early in life, particularly individuals with acommissural aortic valves as they tend to have an aggressive clinical course. Moving forward, it would be valuable to investigate the progression 
of both BAV and UAV over time as the present study lacks long term longitudinal monitoring of disease progression and development.

Although UAV and BAV have distinctly different morphologies, the findings of this study suggest that they represent a continuum of the same disease. Patients with UAV represent a more aggressive phenotype in the spectrum of BAV syndromes, presenting with more severe aortic stenosis at a younger age compared to BAV. Adoption of current recommendations for the management and monitoring of BAV to patients with UAV is a reasonable consideration, with the understanding that both aortic stenosis and aortic complications occur earlier in patients with UAV.

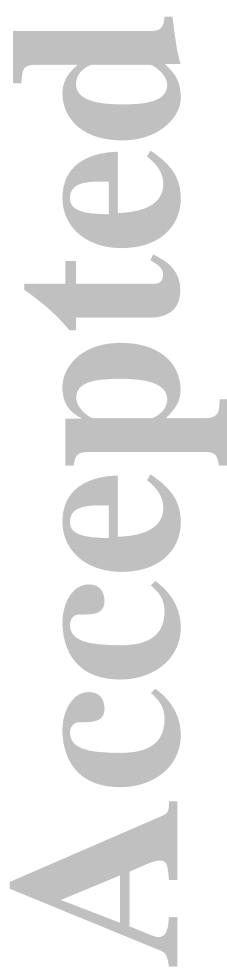




\section{AUTHOR CONTRIBUTIONS:}

All authors participated in the concept/design, data collection, analysis and interpretation, and critical revision of the manuscript.

In addition, Joseph Krepp and Federico Asch participated in drafting of the article.

In addition, Barbara Kroner and Liliana Preiss preformed statistical analysis.
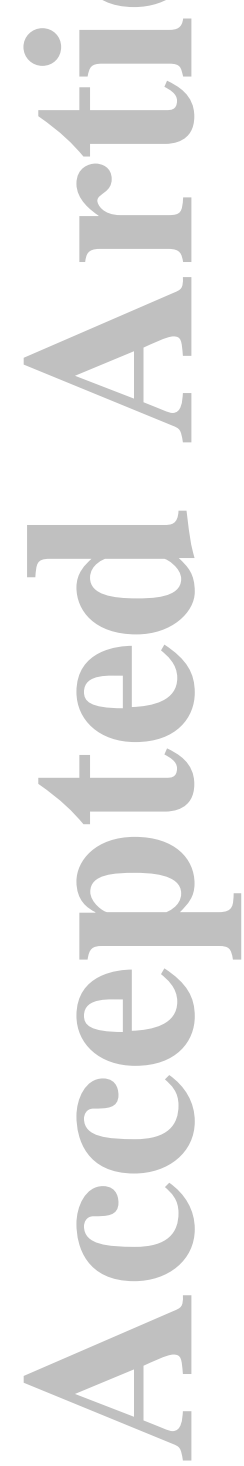


\section{FIGURE CAPTIONS}

Figure 1. Examples of bicuspid and unicuspid aortic valves (BAV and UAV, respectively) and typical ascending aortic enlargement in these conditions. BAV was defined as having two commissures (arrows) and an oval, "football-like" opening (left panel). UAV was defined as a valve with one (or none) commissure and a rounded, "soccer ball-like" opening (center panel). Both examples are shown from a parasternal short axis view. In both conditions, enlargement of the ascending aorta (classically described for BAV) was frequently found, shown in the right panel from a suprasternal notch view (dilated ascending aorta marked in full line, normal descending in dotted line).

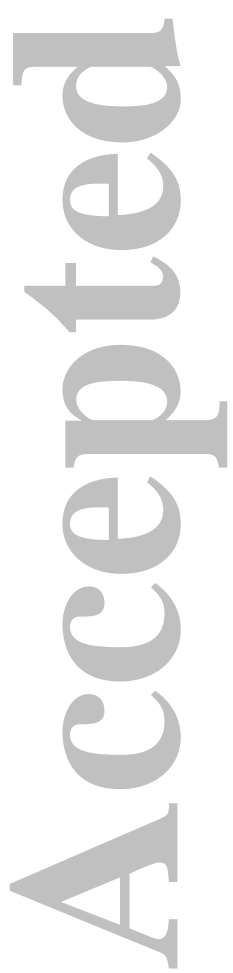




\section{REFERENCES}

1. Mookadam F, Thota VR, Garcia-Lopez AM, Emani UR, Alharthi MS, Zamorano J, Khandheria BK. Unicuspid aortic valve in adults: a systemic review. J Heart Valve Dis. 2010;19:79-85.

2. Roberts WC, Ko JM. Weights of operatively-excised stenotic unicuspid, bicuspid, and tricuspid aortic valves and their relation to age, sex, body mass index, and presence of absence of concomitant coronary artery bypass grafting. Am J Cardiol. 2003;92:10571065 .

3. Debl K, Djavidani B, Buchner S, Poschenrieder F, Heinicke N, Schmid C, Kobuch R, Feuerbach S, Riegger G, Luchner A. Unicuspid aortic valve disease: a magnetic resonance imaging study. Fortschr Röntgenstr. 2008;180:983-987.

4. Cury M, Zeidan F, Lobato AC. Aortic disease in the young: genetic aneurysm syndromes, connective tissue disorders, and familial aortic aneurysms and dissections. Int JVasc Med. 2013:1-7.

5. Bansal A, Arora S, Traub D, Haybron D. Unicuspid aortic valve and aortic arch aneurysm in a patient with Turner syndrome. Asian Cardiovasc Thorac Ann. $2008 ; 16: 266-267$.

6. Brantley HP, Nekkanti R, Anderson CA, Kypson AP. Three-dimensional echocardiographic features of unicuspid aortic valve stenosis correlate with surgical findings. Echocardiography. 2012;29:E204-E207.

7. Ermis N, Bilgi M, Altay H, Erol T. An asymptomatic unicuspid aortic valve with aortic aneurysm and secundum atrial septal defect. Clin Cardiol. 2010;33:E38-39. 
8. Kawase I, Ozaki S, Yamashita H, Uchida S, Nozawa Y, Matsuyama T, Takatoh M, Hagiwara S. Aortic valve reconstruction of unicuspid aortic valve by tricuspidizaiton using autologous pericardium. Ann Thorac Surg. 2012;94:1180-1184.

9. Buchner S, Kobuch R, Luchner A, Debl K. Diagnosis of unicommissural unicuspid aortic valve stenosis by different imaging modalities. J Cardiovasc Med. 011;12:347-348.

10. Chu JW, Picard MH, Agnihotri AK, Fitzsimmons MG. Diagnosis of congenital unicuspid aortic valve in the adult population: the value and limitation of tranesophageal echocardiography. Echocardiography. 2010;27:1107-1112.

11. Roberts WC, Vowels TJ, Ko JM. Natural history of adults with congenitally malformed aortic valves (unicuspid or bicuspid). Medicine. 2012;91:287-308.

12. Fealey ME, Edwards WD, Miller DV, Maleszewski JJ. Unicommissural aortic valves: gross, histological, and immunohistochemical analysis of 52 cases (1978-2008).

Cardiovasc Pathol. 2012;21:324-333.

13. Agnihotri AK, Desai SC, Lai YQ, Fitzsimons MG, Hilgenberg AD, Vlahakes GJ. Two distinct clinical presentations in adult unicuspid aortic valve. J Thorac Cardiovasc Surg. 2006;131:1169-1170.

14. Collins MJ, Butany J, Borger MA, Strauss BH, David TE. Implications of a congenitally abnormal valve: a study of 1025 consecutively excised aortic valves. J Clin Pathol. $2008 ; 61: 530-536$.

15. Eagle KA. GenTAC Consortium. Rationale and design of the National Registry of Genetically Triggered Thoracic Aortic Aneurysms and Cardiovascular Conditions (GenTAC) Registry. Am Heart J.2009;157:319-326. 
16. Kroner BL, Tolunay HE, Basson CT, Pyeritz RE, Holmes KW, Maslen CL, Milewicz DM, Lemaire SA, Hendershot T, Desvigne-Nickens P, Devereux RB, Dietz HC, Song HK, Ringer D, Mitchell M, Weinsaft JW, Ravekes W, Menashe V, Eagle KA. The National Registry of Genetically Triggered Thoracic Aortic Aneurysms and Cardiovascular Conditions (GenTAC): Results from Phase I and Scientific Opportunities in Phase II. Am Heart J. 2011;4:627-632.

17. Asch FM, Yuriditsky E, Prakash SK, Devereux RB, Roman MJ, Weinsaft JW, Weissman G, Weigold WG, Morris SA, Ravekes WJ, Holmes KW, Silberbach M, Milewski K, Kroner BL, Whitworth R, Eagle KA, Weissman NJ: The need for standardized methods for measuring the aorta: Multimodality core lab experience from the GenTAC Registry. JACC Imaging 2016;9:219-226.

18. Zoghbi W, Enriquez-Sarano M, Foster E, Grayburn P, Kraft C, Levine R, Nihoyannopoulos P, Otto C, Quinones M, Rakowski H, Stewart W, Waggoner A, Weissman N. Recommendations for evaluation of the severity of native valvular regurgitation with two-dimensional and Doppler echocardiography. J Am Soc Echocardiog. 2003;16:777-802.

19. Baumgartner H, Hung J, Bermejo J, Chambers J, Evangelista A, Griffin B, Iung B, Otto C, Pellikka P, Quinones M. Echocardiographyic Assessment of valve stenosis: EAE/ASE Recommendations for Clinical Practice. J Am Soc Echocardiog. 2009; 22:123.

20. Michelena H, Desjardins V, Avierinos JF, Russo A, Nkomo V, Sundt T, Pellikka P, Tajik AJ, Enriquez-Sarano M. Natural history of asymptomatic patients with normally 
functioning or minimally dysfunctional bicuspid aortic valve in the community.

Circulation. 2008;2776-2784.

21. Hahn RT, Roman MJ, Mogtader AH, Devereux RB. Association of aortic dilatation with regurgitant, stenotic and functionally normal bicuspid aortic valves. J Am Coll Cardiol. $1992 ; 19: 283-288$.

22. Keane MG, Wiegers SE, Plappert T, Pochettino A, Bavaria JE, Sutton MGSJ. Bicuspid . aortic valves are associated with aortic dilatation out of proportion to coexistent valvular lesions. Circulation. 2000;102:III35-III39.

23. Cripe L, Andelfinger G, Martin LJ, Shooner K, Benson DW. Bicuspid aortic valve is heritable. J Am Coll Cardiol. 2004;44:138-143.

24. Roberts WC, Ko JM. Frequency by decades of unicuspid, bicuspid, and tricuspid aortic valves in adults having isolated aortic valve replacement for aortic stenosis, with or without associated aortic regurgitation. Circulation. 2005;111:920-925.

25. Ichihara T, Gijii G, Sasaki M, Kawaguchi O, Ueda Y. Calcific aortic stenosis and congenital bicuspid aortic valves. Asian Cardiovasc Thorac Ann. 2006;14:210-212.

26. Ucak A, Inan K, Onan B, Temizkan V, Kilicaslan F, Yilmaz AT. Left sinus of Valsalva aneurysm in an adult with congenital unicuspid aortic valve. Int J Cardiol. 2011;149:E21-E23.

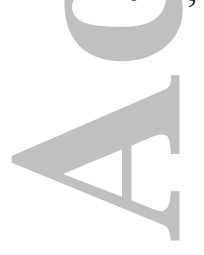




\section{SUPPLEMENTAL MATERIAL}

GenTAC Registry Investigators:

Johns Hopkins University

Williams Ravekes, M.D.

Harry C. Dietz, M.D., Ph.D.

Kathryn W. Holmes, M.D.

Jennifer Habashi, MD

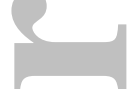

$\underline{\text { University of Texas - Houston }}$

Dianna M. Milewicz, M.D. Ph.D.

Siddharth K. Prakash, M.D., Ph.D

Baylor College of Medicine

Scott A. LeMaire. M.D.

Shaine A. Morris, M.D.

$\underline{\text { Oregon Health \& Science University }}$

Cheryl L Maslen, Ph.D.

Howard K. Song, M.D., Ph.D

G. Michael Silberbach, M.D.

$\underline{\text { University of Pennsylvania }}$

Reed E. Pyeritz, M.D., Ph.D. 
Joseph E. Bavaria M.D.

Karianna Milewski, M.D., Ph.D.

$\underline{\text { Weill Medical College of Cornell University }}$

Richard B. Devereux, M.D., Ph.D.

Jonathan W. Weinsaft, M.D.

Mary J. Roman, M.D.

The Queen's Medical Center

Ralph Shohet, M.D.

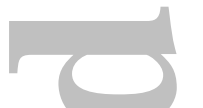

National Institute on Aging

Nazli McDonnell, MD
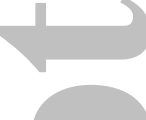

$\underline{\text { MedStar Health Research Institute }}$

Federico M. Asch, M.D.

$\underline{\text { University of Michigan }}$

Kim A. Eagle, M.D.

$\underline{\text { National Heart, Lung, and Blood Institute }}$

H. Eser Tolunay, Ph.D.

Patrice Desvigne-Nickens, M.D. 
National Institute of Arthritis, Musculoskeletal and Skin Diseases

Hung Tseng, PhD

$\underline{\text { RTI International }}$

Barbara L. Kroner, Ph.D.
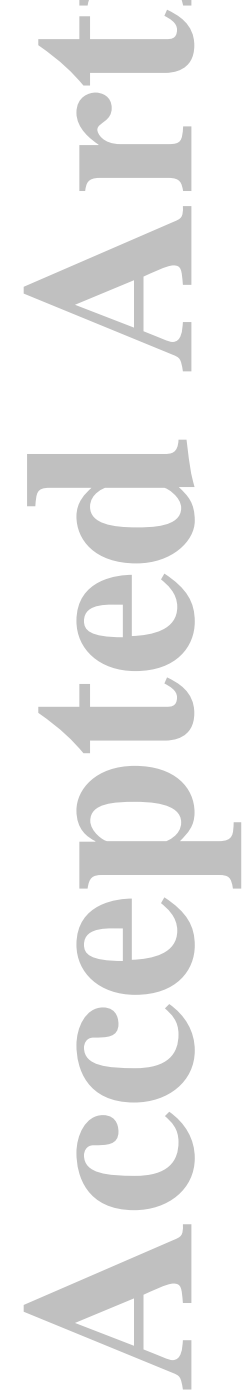


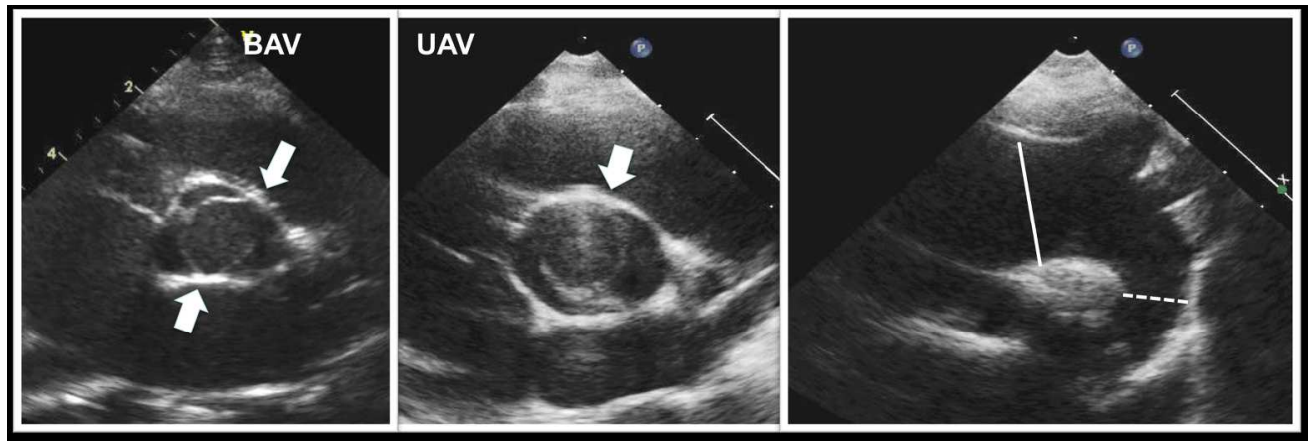

Figure 1. Examples of bicuspid and unicuspid aortic valves (BAV and UAV, respectively) and typical ascending aortic enlargement in these conditions. BAV was defined as having two commissures (arrows) and an oval, "football-like" opening (left panel). UAV was defined as a valve with one (or none) commissure and a rounded, "soccer ball-like" opening (center panel). Both examples are shown from a parasternal short axis view. In both conditions, enlargement of the ascending aorta (classically described for BAV) was frequently found, shown in the right panel from a suprasternal notch view (dilated ascending aorta marked in full line, normal descending in dotted line).

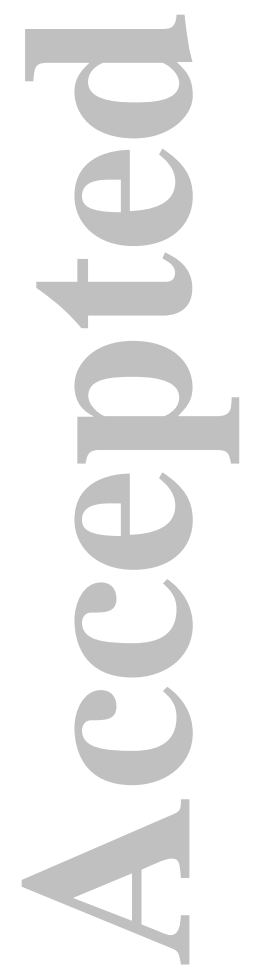

$793 \times 266 \mathrm{~mm}(72 \times 72 \mathrm{DPI})$ 
Table I. Patient Demographics

\begin{tabular}{|c|c|c|c|}
\hline & UAV & BAV & p value \\
\hline Age, years & 15.4 & 15.4 & -- \\
\hline Male (\%) & $70.6 \%$ & $70.6 \%$ & -- \\
\hline Body Surface Area, m² & 1.25 & 1.22 & -- \\
\hline \multicolumn{4}{|l|}{ Aortic Surgeries (n, \%) } \\
\hline Ascending & $1(5.9)$ & $2(11.8)$ & 1.000 \\
\hline Arch and Descending & $0(0.0)$ & $1(5.9)$ & 1.000 \\
\hline Coarctation Repair & $4(23.5)$ & $3(17.6)$ & 1.000 \\
\hline Aortic Dissection (n, \%) & $0(0.0)$ & $1(5.9)$ & 1.000 \\
\hline Family History of Disease & $\mathrm{n}=8$ & $\mathrm{n}=11$ & \\
\hline BAV & 3 & 1 & 0.260 \\
\hline Aortic Coarctation & 1 & 0 & 0.420 \\
\hline Marfan Syndrome & 0 & 1 & 1.000 \\
\hline Aortic Aneurysm & 1 & 2 & 1.000 \\
\hline Aortic Dissection & 0 & 0 & 1.000 \\
\hline
\end{tabular}


Table II. Echocardiographic and Hemodynamic Data

\begin{tabular}{|c|c|c|c|}
\hline Echo Findings & UAV & BAV & p value \\
\hline \multicolumn{4}{|l|}{ AV gradient, mm Hg (mean, SD) } \\
\hline Mean & $22.4(10.6)$ & $10.4(7.6)$ & $<0.001$ \\
\hline Peak & $39.2(17.3)$ & $18.6(15.6)$ & $<0.001$ \\
\hline AVstenosis (n, \%) & & & 0.011 \\
\hline None & $2(11.7)$ & $11(68.75)$ & \\
\hline Mild & $5(29.4)$ & $2(12.5)$ & \\
\hline Moderate or Severe & $10(58.8)$ & $3(18.75)$ & \\
\hline AV regurgitation & & & 0.110 \\
\hline None & $1(5.9)$ & $6(35.3)$ & \\
\hline Trivial or Mild & $13(76.5)$ & $10(58.8)$ & \\
\hline Moderate or Severe & $3(17.6)$ & $1(5.9)$ & \\
\hline AV Degeneration Score $(\text { mean, } S D)^{a}$ & $2.8(1.6)$ & $1.7(1.3)$ & 0.043 \\
\hline Mobility & $1.6(0.6)$ & $0.7(0.6)$ & $<0.001$ \\
\hline Thickness $^{\mathrm{b}}$ & $0.9(0.7)$ & $0.8(0.5)$ & 0.718 \\
\hline Calcification ${ }^{b}$ & $0.3(0.7)$ & $0.2(0.5)$ & 0.608 \\
\hline \multicolumn{4}{|l|}{ Aortic dimensions, cm (mean, SD) } \\
\hline Annulus & $2(0.6)$ & $1.9(0.5)$ & 0.656 \\
\hline Sinus of Valsalva & $2.7(0.8)$ & $2.8(0.9)$ & 0.900 \\
\hline ST Junction & $2.3(0.7)$ & $2.2(1.8)$ & 0.570 \\
\hline Ascending Aorta & $3(1.0)$ & $2.7(0.8)$ & 0.232 \\
\hline Proximal Arch & $2(0.7)$ & $2.1(0.8)$ & 0.757 \\
\hline
\end{tabular}




\begin{tabular}{lccc}
\hline Transverse Arch & $1.6(0.6)$ & $1.8(0.7)$ & 0.487 \\
\hline Isthmus & $1.2(0.4)$ & $1.3(0.4)$ & 0.696 \\
\hline Descending Aorta & $1.3(0.3)$ & $1.6(0.5)$ & 0.140 \\
\hline
\end{tabular}

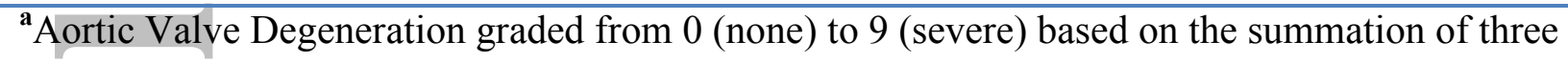
components: leaflet mobility, thickness and calcification

${ }^{\mathrm{b}}$ Graded on a scale of 0 (normal), 1 (mildly abnormal), 2 (moderately abnormal), 3 (severely abnormal)
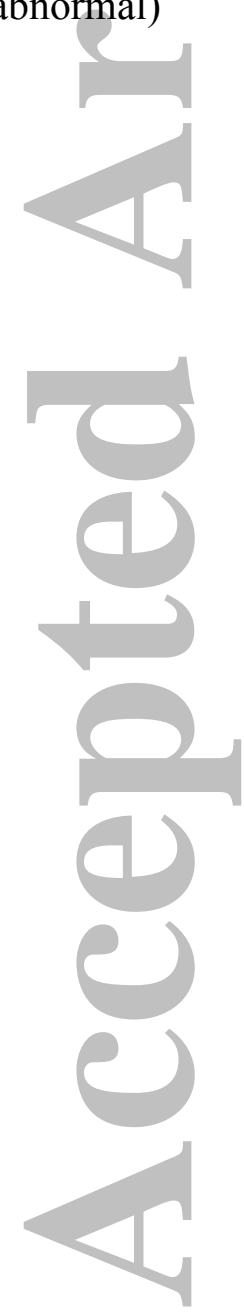
Table III. Comparison of Subjects with Aortic Stenosis

\begin{tabular}{|c|c|c|c|}
\hline Variable & UAV $(n=15)$ & $\operatorname{BAV}(n=5)$ & p value \\
\hline Age, years (mean, SD) & $15.3(15.3)$ & $22.6(20.8)$ & 0.406 \\
\hline \multicolumn{4}{|l|}{ AV gradient, mm Hg (mean, SD) } \\
\hline Mean & $24.3(9.6)$ & $19.4(7.6)$ & 0.312 \\
\hline Peak & $42.6(15.2)$ & $37.2(16.5)$ & 0.509 \\
\hline Indexed AVA, $\mathrm{cm}^{2} / \mathrm{m}^{2}$ (mean, SD) & $0.67(0.12)$ & $0.90(0.22)$ & 0.011 \\
\hline AV Degeneration Score (mean, SD) ${ }^{\mathrm{a}}$ & $3.1(1.5)$ & $2.4(1.7)$ & 0.410 \\
\hline Mobility $^{b}$ & $1.7(0.5)$ & $0.8(0.8)$ & 0.005 \\
\hline Thickness $^{b}$ & $1(0.7)$ & $1.2(0.4)$ & 0.537 \\
\hline Calcification $^{b}$ & $0.3(0.7)$ & $0.4(0.9)$ & 0.868 \\
\hline
\end{tabular}

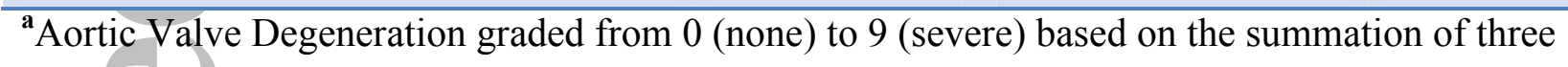
components: leaflet mobility, thickness and calcification

${ }^{\mathrm{b}}$ Graded on a scale of 0 (normal), 1 (mildly abnormal), 2 (moderately abnormal), 3 (severely abnormal) 